\title{
Using Adult Drug Efficacy Data to Aid in Interpretation of Underpowered Pediatric Studies
}

\author{
Kathryn M. Haider, MDㄹ David K. Wallace, MD, MPH ${ }^{1}$
}

1 Department of Ophthalmology, Indiana University School of Medicine, Riley Hospital for Children, Indianapolis

Ophthalmia neonatorum (ON) is an infectious condition of the ocular conjunctiva that occurs in infants younger than 1 month. Infants are thought to have increased levels of exposure to pathogens during the birthing process, and it is believed that many bacterial or viral infections are passed from mother to infant in the birth canal. In addition, neonates are believed to be more susceptible to acquiring infections owing to reduced immunity.

When treating $\mathrm{ON}$, it is important to consider both the most common bacterial pathogens and the pathogens capable of causing the worst complications. Although uncommon, infections of Neisseria gonorrhoeae have been known to cause corneal perforation and meningitis, whereas Chlamydia trachomatis infections have led to permanent vision loss and life-threatening pneumonia. It is also prudent to consider the safety profile of the medication used for the treatment of ON. Silver nitrate was very effective at reducing $\mathrm{ON}$, but its use was discontinued because of toxic effects. Ideally, antibiotic coverage should be well tolerated, broad spectrum, and cover the worst offenders.

For many reasons, most medications used to treat ON were being used off-label. The US Food and Drug Administration (FDA) requested that ON be studied to determine whether the therapeutic agents being used were effective against ON. The agents studied were already approved for the treatment of

This is the author's manuscript of the work published in final edited form as:

Haider, K. M., \& Wallace, D. K. (2021). Using Adult Drug Efficacy Data to Aid in Interpretation of Underpowered Pediatric Studies. JAMA Ophthalmology, 139(2), 217-218. https://doi.org/10.1001/jamaophthalmol.2020.5557 
bacterial conjunctivitis $(\mathrm{BC})$ in older children and adults. Three prospective studies ${ }^{1-3}$ were completed by drug companies on 3 different fluoroquinolone agents, but all were underpowered to prove efficacy.

In this issue of JAMA Ophthalmology, Sun et al ${ }^{4}$ pose the question of whether $\mathrm{ON}$ in neonates and $\mathrm{BC}$ in older children and adults are similar in terms of the cause of the disease and the therapeutic response. If they are, then it may be reasonable to extrapolate the efficacy data from the studies of older children and adults to the neonatal population in an effort to support FDA approval for those medications for the treatment of $\mathrm{ON}$. The authors compared the 3 prospective studies of fluoroquinolones used to treat ON with many recent and comparative studies of the same 3 fluoroquinolones used to treat $\mathrm{BC}$ in older children and adults. The authors focused on efficacy and did not address the safety profile of these medications.

The authors found that the bacterial pathogens in both the ON neonatal group and the BC group of older children and adults were similar. They also found that the clinical trial cure rates, both clinical course and treatment response, were similar between the 2 groups. They concluded that, because the groups were similar, extrapolation of clinical efficiency seemed reasonable. Based on that conclusion, the 3 drugs that were previously evaluated in underpowered studies were approved by the FDA for treatment of ON.

The rate of off-label medication use is $40 \%$ in the pediatric population and nearly $90 \%$ in the neonatal population. ${ }^{5,6}$ There are many challenges to clinical trial recruitment in the pediatric population, making it difficult to obtain evidence-based research to guide clinical practice. We commend the authors for making use of previous data in new ways to address the needs of this vulnerable patient population. A particular strength of their study was that they chose only high-quality, randomized, double-masked clinical trials for comparison. They compared trials using the same therapeutic drugs prescribed in a 
similar way. When possible, they looked for end points that were predefined, quantifiable, and objective.

A key question is whether neonatal $\mathrm{ON}$ and $\mathrm{BC}$ in older children and adults are similar in terms of the cause of the disease and the therapeutic response. This study supports the premise that the 2 diseases have similar bacterial pathogens and a similar clinical course. The point estimates for efficacy from all 3 ON studies were within the $95 \% \mathrm{Cl}$ of the studies of older children and adults. However, each of these point estimates represents data from only 1 study, and as such, we do not know that they represent the true efficacy values for the neonatal population. If the true value falls outside the range of the $95 \% \mathrm{Cl}$ of the studies of older children and adults, then this approach could be misleading. Still, it is reassuring that all 3 fluoroquinolones fall within the $95 \%$ Cls of the studies of older children and adults.

The authors included a detailed list of cultured organisms and microbial resolution. It is reassuring that there is considerable overlap between $\mathrm{ON}$ and $\mathrm{BC}$. Their table does not report the relative prevalence, the likelihood of being a pathogen (vs being a contaminant), or antibiotic susceptibility. We would be concerned, for example, if a particular organism is much more prevalent in ON than in $\mathrm{BC}$ in older children and adults, is more likely to be a pathogen instead of a contaminant, and is less susceptible to a fluoroquinolone. There were no cases of Neisseria gonorrhoeae or chlamydia, which are important ON pathogens.

With reductions in the incidence of chlamydia and gonorrhea over time, the most common bacterial pathogens in $\mathrm{ON}$ are closer in presentation and clinical course to those in $\mathrm{BC}$ in older children and adults. However, the incidence of these rare pathogens is not zero. It remains important to keep chlamydia, Neisseria gonorrhoeae, and herpes simplex virus at the top of the differential because these pathogens can produce long-lasting ocular and systemic complications if not recognized and treated promptly. 
When faced with ON, we always consider a wide differential diagnosis and will perform a culture on chocolate (Neisseria gonorrhoeae) and blood (bacteria) agar, Gram stain for Neisseria gonorrhoeae, and perform a polymerase chain reaction for chlamydia and a viral polymerase chain reaction for herpes simplex virus. We consult pediatricians to systemically assess the infant, looking for septicemia, pneumonia, and encephalitis. We typically treat for a minimum of 7 days, depending on the clinical presentation. In severe cases, we consider switching to fortified antibiotics if there is not a clinical response after 24 to 48 hours of treatment.

We applaud the authors for their innovative approach to studying the effectiveness of these ophthalmic medications in neonates. Their methods have the potential to be applied to other pediatric diseases, improving our understanding of the effects of medications on infants and children and informing the FDA as they make decisions on labeling.

Corresponding Author: Kathryn M. Haider, MD, Department of Ophthalmology, Indiana University School of Medicine, 1160 W Michigan St, Indianapolis, IN 46202 (khaider@iupui.edu).

Published Online: December 17, 2020. doi:10.1001/jamaophthalmol.2020.5557

Conflict of Interest Disclosures: Dr Wallace reported receiving grants from the National Eye Institute outside the submitted work. No other disclosures were reported. 


\section{References}

1. Chambers WA. Deputy Division Director review of NDA 19-992 supplement 17. US Food and Drug Administration. Accessed June 6, 2020. https://www.fda.gov/media/138599/download

2. Chambers WA. Deputy Division Director review of NDA 21-598. US Food and Drug Administration. Accessed June 6, 2020. https://www.fda.gov/media/104593/download

3. Chambers WA. Deputy Division Director review of NDA 21-493/S-009. US Food and Drug Administration. Accessed June 6, 2020. https://www.fda.gov/media/130102/download

4. Sun $\mathrm{H}$, Temek JW, McCune SK, Chambers WA. Using historical data to support approval of drug products for ophthalmia neonatorum: innovative approach toward evidence-based medicine. JAMA Ophthalmol. Published online December 17, 2020. doi:10.1001/jamaophthalmol.2020.5558

5. Sachs AN, Avant D, Lee CS, Rodriguez W, Murphy MD. Pediatric information in drug product labeling. JAMA. 2012;307(18):1914-1915. doi:10.1001/jama.2012.3435

6. Sun H, Temeck JW, Chambers W, Perkins G, Bonnel R, Murphy D. Extrapolation of efficacy in pediatric drug development and evidence-based medicine: progress and lessons learned. Ther Innov Regul Sci. 2017;2017:1-7. 